\title{
Manual assisted cognitive behaviour therapy is as effective as treatment as usual for deliberate self harm, but is more cost effective
}

Tyrer P, Thompson S, Schmidt U, et al. Randomized controlled trial of a brief cognitive behaviour therapy versus treatment as usual in recurrent deliberate self-harm: the POPMACT study. Psychol Med 2003;33:969-76; and Byford S, Knapp M, Greenshields J, et al. Cost-effectiveness of brief cognitive behaviour therapy versus treatment as usual in recurrent deliberate self-harm: a decision-making approach. Psychol Med 2003;33:977-86.

\section{Q Is manual assisted cognitive behaviour therapy more effective and more cost effective than treatment as usual in people with recurrent episodes of deliberate self harm?}

METHODS

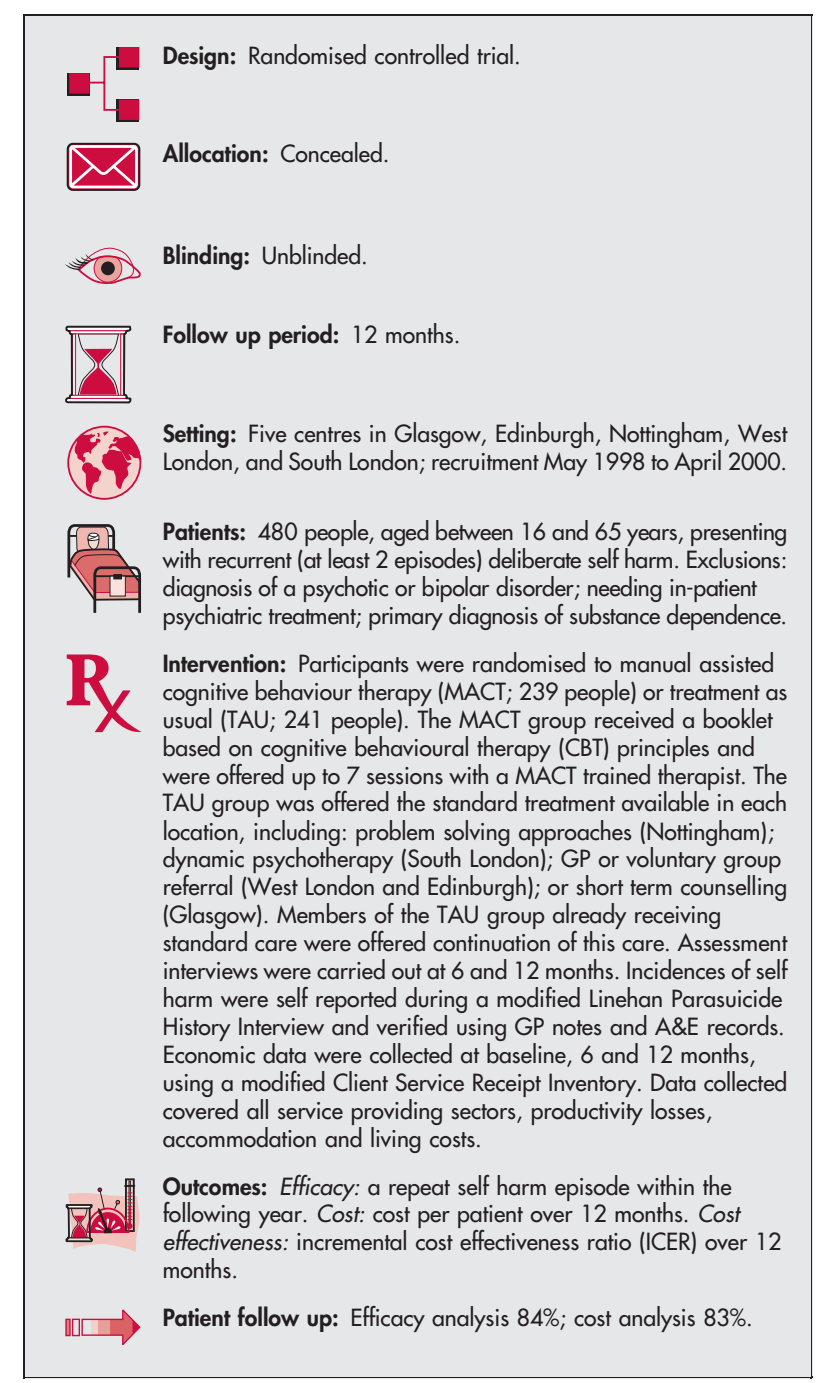

\section{MAIN RESULTS}

Efficacy: at 12 months, the proportion of participants repeating deliberate self harm was not significantly different between groups (AR 39\% with MACT $v 46 \%$ with TAU; OR $0.78,95 \%$ CI 0.53 to 1.14 ; analysis not by intention to treat). Cost: MACT was significantly

For correspondence: Professor Peter Tyrer, Imperial College, St Dunstan's Road, London, UK

Source of funding: The Medical Research Council, UK. cheaper than TAU over 6 months, but did not maintain significance over 12 months ( 6 month mean difference in cost per patient: $-£ 897$, $95 \% \mathrm{CI}-£ 1747$ to $-£ 48 ; 12$ month mean difference in cost per patient: $-£ 838,95 \%$ CI $-£ 2142$ to $£ 466$ ). Cost effectiveness: the ICER was $-£ 120$ per $1 \%$ reduction in the proportion of participants with a repeat self harm episode. The likelihood that MACT is more cost effective than TAU, based on cost effectiveness acceptability curves, is over $90 \%$.

\section{CONCLUSIONS}

MACT is as effective as usual treatment in reducing recurrences of deliberate self harm, however, it is more cost effective.

\section{NOTES}

Authors note that there were sociodemographic differences between participants included and those excluded from the economic analysis due to missing economic data. There was a centre bias among excluded participants, and they were younger and had cost less in the 6 months prior to baseline. This may affect generaliseability of results.

\section{Commentary}

These companion papers by Tyrer et al and Byford et al are excellent models of psychiatric research which have produced sobering and disappointing results in terms of effecting a reduction in deliberate self harm. However, it would be reassuring to health administrators that there may be a reduction in health care expenditure with the manual assisted cognitive behaviour therapy.

If such a well designed study cannot demonstrate an improved clinical outcome, a finding which is consistent with the majority of previous research, questions arise not only about the wisdom of pursuing further such studies, but also whether the correct issues are being addressed.

Consider the subjects of the study: they have had a previous episode, and therefore they would be a more homogeneous group; they did not require in-patient care, so they may be less ill than some seen in practice; and they did not have a psychotic disorder or bipolar disorder, or a primary diagnosis of substance dependence, therefore precluding a further substantial group seen in practice. And yet they are a patient group united by deliberate self harm, a behaviour related to many disparate stressors. Is such a behaviour sufficient to form a cohesive group worthy of such intensive study?

In 1828 George Burrows' wrote that "The medical treatment of the propensity to suicide, whether prophylactic or therapeutic, differs not from that which is applicable in cases of ordinary insanity." This advice to address the underlying condition appears to have been overlooked in our attempts to demonstrate our ability to influence a behaviour.

It is doubtful whether a better study design focusing on deliberate self harm could be implemented. Indeed, perhaps the most important implication from this study is that future psychiatric research should focus on the antecedents of deliberate self harm, that is those conditions which are associated with it rather than the behaviour itself. Robert Goldney MB, BS, MD, FRANZCP, FRC Psych Professor of Psychiatry, University of Adelaide, Australia 1 Burrows, GM. Commentaries on the causes, forms, symptoms, and treatment, moral and medical, of insanity. London: Thomas and George Underwood, 1828 